\title{
Laboratory Test Numeric Result in Standard Unit
}

National Cancer Institute

\section{Source}

National Cancer Institute. Laboratory Test Numeric Result in Standard Unit. NCI

Thesaurus. Code C83320.

The numerical identifier of the laboratory test result in standard units. 